\title{
The hard X-ray/soft $\gamma$ ray sky as seen with INTEGRAL/SPI: point sources and diffuse emission
}

\section{Bouchet ${ }^{* \dagger}$ E. Jourdain, J.-P. Roques,}

Université de Toulouse, UPS-OMP, IRAP, Toulouse, France

CNRS, IRAP, 9 Av. colonel Roche, BP 44346, F-31028 Toulouse cedex 4, France

E-mail: bouchetecesr.fr

\section{A. Strong, R. Diehl,}

Max-Planck-Institut für extraterrestrische Physik, Postfach 1603, 85740 Garching, Germany

\begin{abstract}
With more than six years of operation, the spectrometer SPI, onboard the INTEGRAL observatory, gives us a unique opportunity to map the hard X-ray sky, $20 \mathrm{keV}-2.5 \mathrm{MeV}$, for both point sources and "diffuse" emission. We present an all-sky census of the high energy sources with SPI. Taking advantage of $\sim 10^{8}$ s of observation time, we have measured a detailed ridge spectrum and constrained the spatial distribution of the emission. The interstellar part of the ridge emission can be explained by inverse-Compton scattering from relativistic electrons on the cosmic microwave background and the Galactic infrared and optical radiation fields. The spectrum is compared with predictions from GALPROP modelling.
\end{abstract}

8th INTEGRAL Workshop "The Restless Gamma-ray Universe" - Integral2010,

September 27-30, 2010

Dublin Ireland

* Speaker.

${ }^{\dagger}$ A footnote may follow. 


\section{INSTRUMENTS, OBSERVATIONS AND DATA ANALYSIS METHOD}

SPI is a spectrometer which is equipped with an imaging system sensitive both to point sources and extended/diffuse emission. Its atypical coded mask imaging system has a field of view of $30^{\circ}$ and 19 Ge detectors ([1]; ; [2]). Because of this small number of detectors, SPI's imaging capability relies on a specific observational strategy, which is based on a so-called dithering procedure [3]. In consequence, to process a large amount of data and to extract a maximum information, enhanced data-reduction software have been developed to better handle source variability and background variations, and to process all the data simultaneously.

Public data recorded between February 2003 and January 2009 with the INTEGRAL/SPI instrument are analyzed. This results in $\sim 1.1 \times 10^{8}$ s observation time and $\sim 39000$ exposures or pointings, excluding data contaminated by solar flares or the radiation belts.

\subsection{Instumental background modelling}

The SPI background pattern (i.e. relative count rates of the detectors) is relatively stable between Ge detector annealings (performed twice a year), but its intensity varies. However, its intensity is quite stable on a $<6$-hour timescale. In the present analysis, we compute the background pattern per period between two detector annealings ( $\sim 6$ months) allowing its intensity to be variable on a $\sim 6$-hour timescale.

\subsection{Better description of sources variability}

Many sources exhibit variability on time scales ranging from one exposure ( $\sim 2000 \mathrm{~s})$ to years. For a given source, it is necessary to divide its total observation period into smaller meaningful time intervals ("time bins") where it can be considered to have a constant flux within the error bars. An algorithm, based on signal segmentation [П], has been developed to achieve this task. It can also handle input information on source variability from other experiments, for example INTEGRAL/IBIS.

\subsection{Software to process all the data simultaneously}

Due to the atypical imaging system, it is imperative to process simultaneously a maximum of exposures (so-called mosaicking of images is not possible with SPI) to probe the lowest flux levels (for example the "diffuse" emission). However, spatial and temporal description of all sources (position and variation in time) requires a huge number of parameters. Many enhancements have been made at the level of the mathematical algorithms in order to be able to process this large amount of data and to compute the solution (up to one million unknowns) and the associated error bars ([5]; [6]). In short, intensities in the source "time bins" and background intensity variations are fitted to the data (through the response matrix) to maximize the likelihood of the data as described in [8].

\section{Hard X-ray/soft $\gamma$-ray surveys - sources census}

The first hard X-ray/soft $\gamma$-ray survey with a dedicated instrument was performed in the early 90's : The GRANAT/SIGMA telescope detected 37 sources in the $35-1300 \mathrm{keV}$ band during its 
8 years livetime [9]. Subsequent observations with CGRO observatory and RXTE increased the number of sources to $\sim 120$ in the early 2000's. A new census of sources is one of the primary task of the INTEGRAL observatory.

\subsection{Survey/census up to $\sim 100 \mathrm{keV}$}

Below $\sim 100 \mathrm{keV}$, angular resolution is crucial for the survey, especially in crowded regions such as the Galactic centre, hence the imager INTEGRAL/IBIS is the preferred instrument. The 4th IBIS catalogue [10] takes an inventory of more than 700 sources in the $17-100 \mathrm{keV}$ range while 521 objects are detected in the 17-60 keV time averaged map [11]. An hard X-ray survey was also performed with SWIFT/BAT since 2005 and reported 1256 source detections [12].

\subsection{Survey/census above $100 \mathrm{keV}$}

Concerning the survey/census of sources emitting above $100 \mathrm{keV}$, GRANAT/SIGMA identified 5 black-holes candidates plus the Crab Nebula. An INTEGRAL/IBIS census based on 20032005 observations [13] revealed 49 sources in the 100-150 keV band, 14 of them still emitting in the 150-300 keV band. INTEGRAL/SPI is the most sensitive intrument above $\sim 150 \mathrm{keV}$ which is currently in operation and hence is the relevant instrument to perform this high energy survey. Several catalogues, based on source time-averaged emission, have been issued ([14]; [8]). The latest one [6] contains 270, 129, 68 and 30 sources respectively in the 25-50, 50-100, 100-200 and 200-600 keV band.

\subsection{Light curves, spectra and high-energy emission}

Derived products from the INTEGRAL/SPI survey are light curves and spectra for all the detected sources. The spectra are available, among other uses, for statistical studies of source populations ( extragalactic, binary systems containing a neutron star or a black hole candidate) and to complete the data obtained at lower energies by INTEGRAL. Figure 1 shows the mean-time averaged spectrum of the radio galaxy Centaurus A, the black-hole system Cygnus X-1 and the neutrons star system GS1826-24.

In the $100 \mathrm{keV}$ region, reflection, thermal and non-thermal emissions co-exist and the sensitivity of INTEGRAL/SPI is usefull to study them. In particular, the study of these spectra revealed that some sources display a high energy tail above $\sim 150 \mathrm{keV}$, whatever the object class. Hence thermal and non-thermal mechanisms may exist in many types of sources (fig. 1) 15].

\section{INTEGRAL/SPI all-sky view of the Milky-Way}

In the early 1990's, the hard X-ray/ soft- $\gamma$-ray emission of the Milky-Way was difficult to interpret and separate into point-sources emission and "diffuse" interstellar emission, due in particular to the inadequate angular resolution and sensitivity of the instruments of the previous decade. While "diffuse" interstellar emission was expected, its intensity and spatial distribution were difficult to estimate. The first step towards improving our knowledge of this emission was done by the SIGMA/GRANAT imager, which for the first time resolved point-sources. Then, observations combining OSSE/CGRO and SIGMA/GRANAT indicated that at least $50 \%$ of the hard X-ray/soft$\gamma$-ray emission is due to point sources. However, it was expected that point sources can account for 

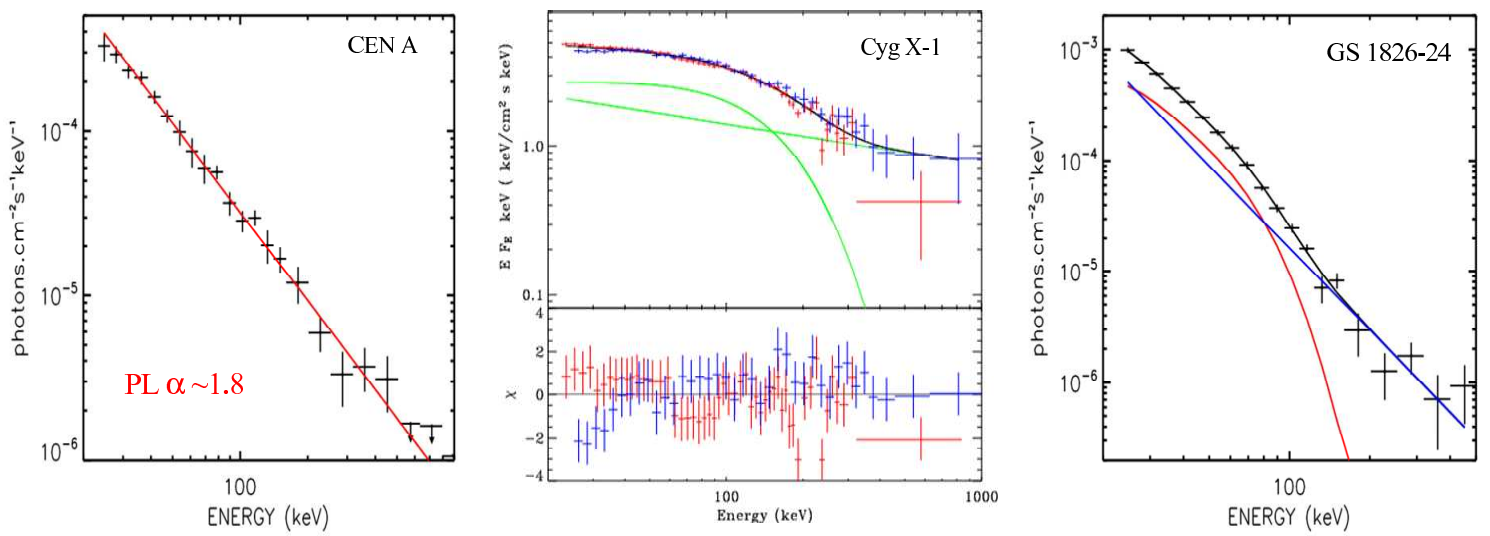

Figure 1: Left: Mean time averaged spectrum of CEN A (Extragalactic) fitted with a power law of index 1.8 [15]. Cyg X-1(Black hole candidate system)(Middle) [33] spectrum modelled with a Comptonization emission law (COMPPS) plus power law and (Right) GS 1826-24 (Neutrons star system) averaged spectrum fitted with a Comptonization law (COMPTT with $\mathrm{kT} \sim 17 \mathrm{keV}$ ) law plus a power law of index $\sim 1.5$ [15].

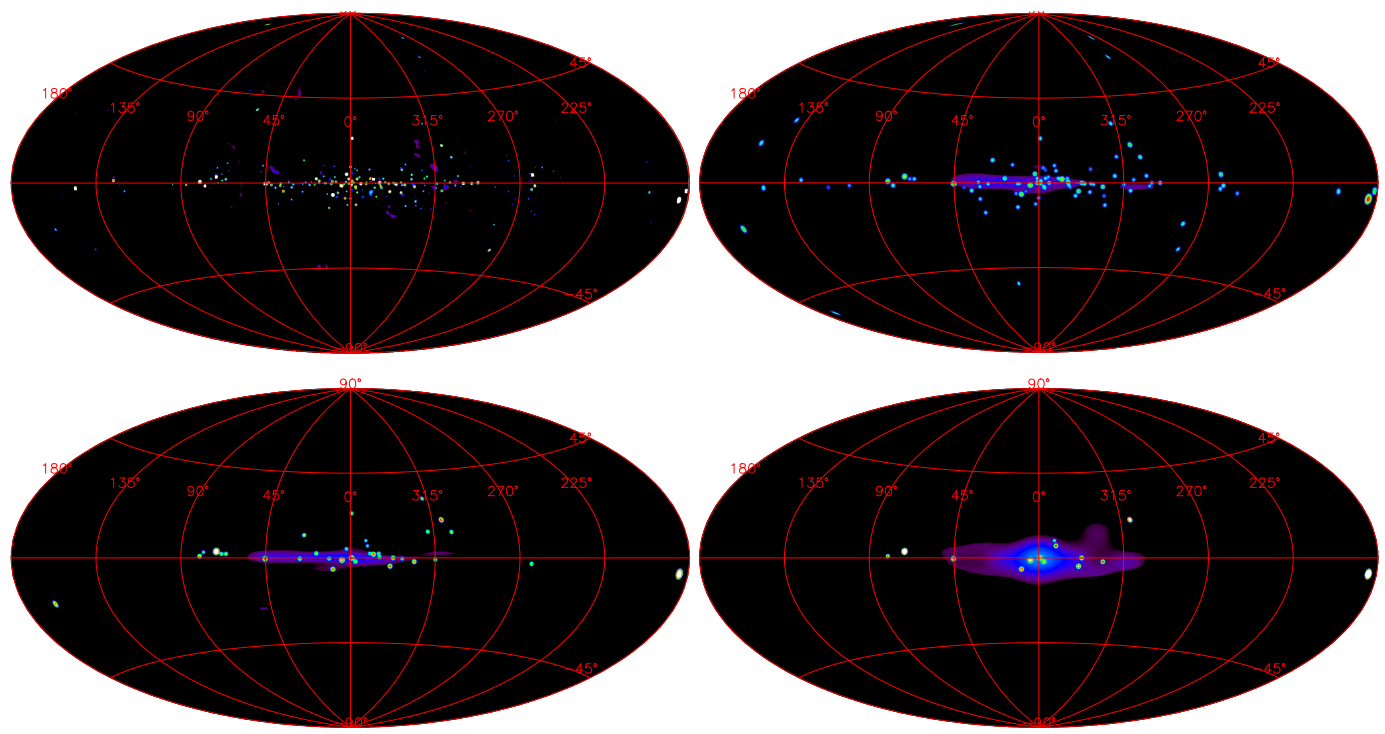

Figure 2: INTEGRAL/SPI all-sky view of the Milky-Way [8]. Top -left : 25-50 keV, top-right : $50-100$ $\mathrm{keV}$, bottom-left : $100-200 \mathrm{keV}$ and bottom-right : $200-600 \mathrm{keV}$

a larger fraction, due in particular to the limited sensitivity of SIGMA/GRANAT. Several measurements followed using RXTE and OSSE, but there was not really much progress.

One of the earlier results obtained with INTEGRAL was that the point sources dominate the Milky-way emission in the 20-200 keV band while "diffuse" interstellar emission is weaker than previously reported [16]. Another main point concerns the annihilation radiation which appears to dominate the sky above $\sim 250 \mathrm{keV}$ and reaches its maximum emission in the narrow emission line at $511 \mathrm{keV}$ [8]. Figure 2 shows the sky seen with INTEGRAL/SPI in several energy bands including both sources and "diffuse" emission. 


\subsection{Diffuse measurements with INTEGRAL below $100 \mathrm{keV}$ and clues to its origin}

Figure 3 shows the "diffuse" (or Galactic Ridge X-ray Emission (GRXE)) measured by both SPI and IBIS onboard INTEGRAL observatory. Based on these data and those of RXTE/PCA at lower energies, [17] and [18] argue convincingly that a population of stellar sources comprised mainly of cataclysmic variables (CVs) and intermediate polars explains a large fraction of the diffuse emission below $50 \mathrm{keV}$. The main characteristics of this GRXE emission is that its spatial distribution follows the NIR/DIRBE 3.5-4.9 $\mu$ map while its spectrum presents a spectral cutoff at 30-50 keV typical of the mean averaged spectrum of intermediate polars and a total luminosity of $4-6 \times 10^{37} \mathrm{erg} . \mathrm{s}^{-1}$. The instruments currently in operation are not sensitive enough to resolve these sources.

\subsection{Interstellar diffuse emission measurements with INTEGRAL/SPI}

Interstellar emission arises from gas in our Galaxy, cosmic-ray constituents (p, He, e-,...) and the interstellar radiation field, through various interactions like inverse Compton, bremsstrahlung, synchroton, $\ldots$ as well as radiactive nuclei decay $\left({ }^{10} \mathrm{Be},{ }^{10,11} \mathrm{~B}, \mathrm{e} \ldots\right)$. For this purpose, Galactic propagation codes such as GALPROP (e.g. [19] and references therein) have been developed to gain insights into the high-energy interstellar medium and to derive the cosmic-ray luminosity and energy budget of the Milky Way [19]. The model can be applied to many experiments like FERMI, WMAP, PLANCK, INTEGRAL and MILAGRO. The code has been used here to predict the Xray $/ \gamma$-ray intensity and spatial distribution of the interstellar "diffuse" emission.

INTEGRAL/SPI is the relevant instrument to measure the "diffuse" spectrum from $20 \mathrm{keV}$ up to few MeV. We can use "diffuse" emission spatial morphology to constrain the electron spectrum and distribution in the Galaxy. We have found that the spatial morphology of the diffuse emission can be well modelled with a combination IC, NIR $4.9 \mu$ maps plus a bulge [6]. The IC map is the emission (spatial distribution and intensity) predicted by the GALPROP code for the inverseCompton emission process.

The $4.9 \mu$ map corresponds to the unresolved sources population as expected and discussed previously (section 3.1) while the bulge (a combination of $3^{\circ}$ and $12^{\circ}$ Gaussians [21]; [20]) describes the $511 \mathrm{keV}$ line as well as the annihilation radiation continuum (positronium) distribution. The statistic above $600 \mathrm{keV}$ does not allow to derive a more sophisticated spatial description, but an IC map provides a good tracer. All this are discussed in more detailled and developped in [6].

The INTEGRAL/SPI "diffuse" spectrum from $20 \mathrm{keV}$ to $2.5 \mathrm{MeV}$ is shown on fig. 3. This spectrum corresponds to the central radian of our Galaxy $\left(|l|<30^{\circ},|b|<15^{\circ}\right)$. It is composed of several components:

- Annihilation radiation spectrum : For the bulge, the line flux is $0.91 \times 10^{-3} \mathrm{ph} \mathrm{cm}^{-2} \mathrm{~s}^{-1}$ consistent with the published results ([8]; [21]). The positronium fraction is around $100 \%$. Detailed spectroscopy has been made by [22], [23]. The possible source of positrons is a key point, discussed e.g. by [24].

- Galactic radioactivities: The $1.8 \mathrm{MeV}$ total flux is about $3.6 \times 10^{-4} \mathrm{ph} \mathrm{cm}^{-2} \mathrm{~s}^{-1}$ for the region delimited by $|l|<40^{\circ},|b|<40^{\circ}$ about $4.3 \times 10^{-4} \mathrm{ph} \mathrm{cm}^{-2} \mathrm{~s}^{-1}$. This value is comparable to the $4 \times 10^{-4} \mathrm{ph} \mathrm{cm}^{-2} \mathrm{~s}^{-1}$ found by [25]. The ratio ${ }^{60} \mathrm{Fe} /{ }^{26} \mathrm{Al}$ provides a test for 

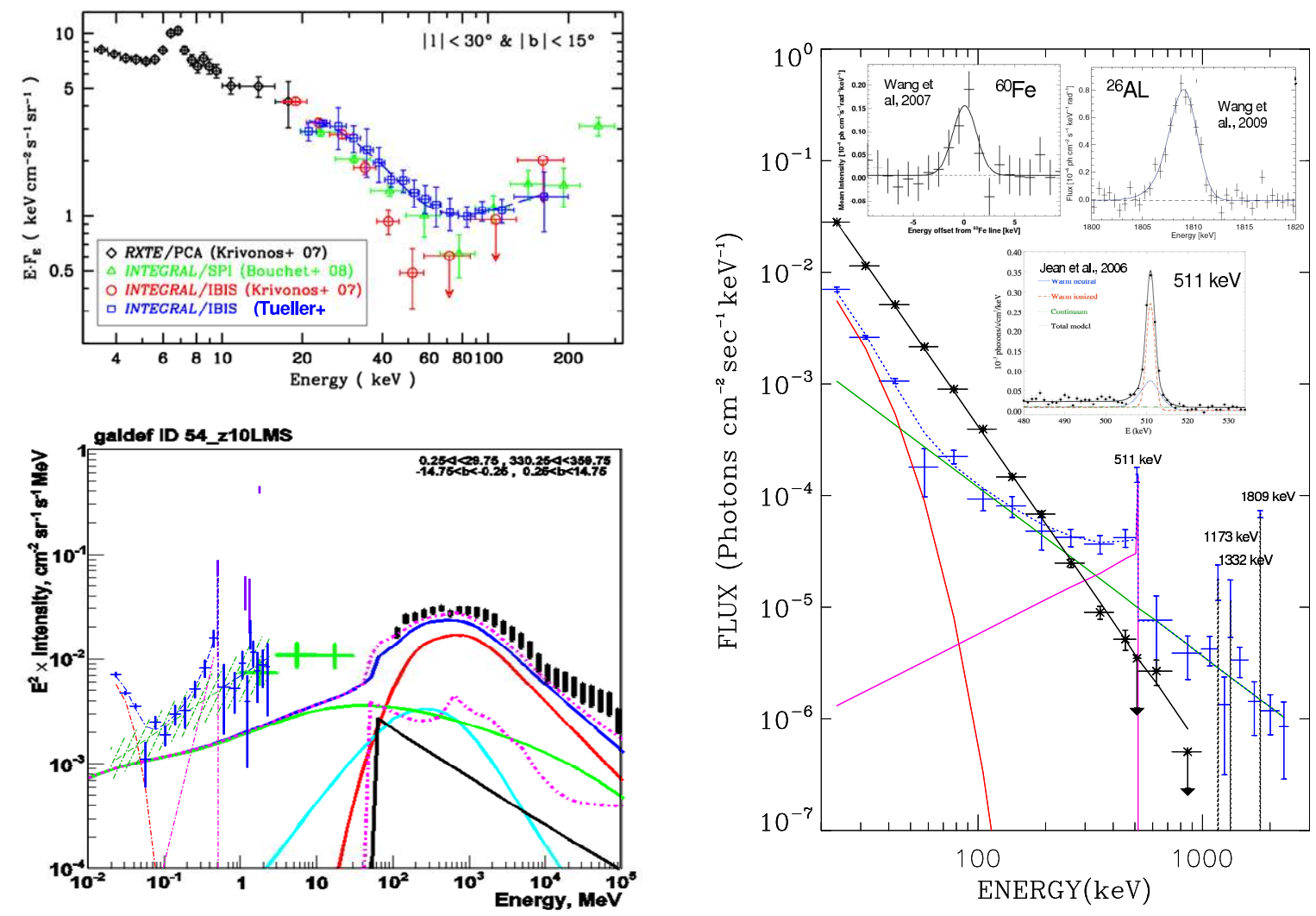

Figure 3: (Top left) For the region $|l|<30^{\circ}$ and $|b|<15^{\circ}$ - Top-left: Comparison of the spectrum obtained by GRXE with INTEGRAL/IBIS, INTEGRAL/SPI and RXTE/PCA. Figure is from [29] - Left: INTEGRAL/SPI Spectra of the different emission components Black: total of resolved source emission - Blue: total diffuse emission -Cyan: annihilation radiation spectrum (line + positronium)- Red: Emission of "unresolved" sources. Green : deduced interstellar particle interactions. (Bottom-left) : Broad band diffuse emission spectra (preliminary) Blue data points are for SPI $\left({ }^{26} \mathrm{Al}\right.$ and ${ }^{60} \mathrm{Fe}$ lines are shown in violet), light green data points are for COMPTEL, and black points are for Fermi. Green dashed : SPI deduced interstellar particle interactions. The blue line is the total emission as calculated with the GALPROP code. $\pi^{0}$ decay, red solid line; IC (optical), green long-dashed line; IC (IR), green short-dash line; IC (CMB), green dotted line; total IC, green solid line; bremsstrahlung, cyan solid line.

nucleosynthesis models. The value obtained in the present analysis, $\sim 0.2$, is in good agreement with those obtained with RHESSI [26] and earlier measurements by INTEGRAL/SPI [27, 28].

- Unresolved source population [18]: The spectrum is well described by an exponential cutoff spectrum with a flux at $50 \mathrm{keV}$ of $\sim 1 \times 10^{-4} \mathrm{ph} \mathrm{cm}^{-2} \mathrm{~s}^{-1} \mathrm{keV}^{-1}$, a cutoff energy of $\sim 8$ $\mathrm{keV}$. More generally, the low energy part below $100 \mathrm{keV}$ is compatible with results obtained from INTEGRAL/IBIS data analysis [18, 29]. This part is linked to the population synthesis.

- Cosmic-ray interactions (modelled with the GALPROP code) : The "diffuse" Galactic ridge spectrum is compatible with earlier results [8]. The "diffuse" emission spectrum obtained with the present analysis confirms the results reported in ([8, 30] with more details. We 
found a diffuse flux around $1 \mathrm{MeV}$ compatible with the COMPTEL/CGRO measurements. The "diffuse" emission is estimated to represent one tenth of the total emission below 100 $\mathrm{keV}$ and one third in 100-300 keV band with a spectrum (apart from positronium) fitted by a power law of index $\sim 1.5$, and a flux at $100 \mathrm{keV}$ of $1.0 \times 10^{-4} \mathrm{ph} \mathrm{cm}^{-2} \mathrm{~s}^{-1} \mathrm{keV}^{-1}$. The hard $\mathrm{X}$-ray continuum is quite consistent with the predictions made with the GALPROP code, in terms of spectral index and spatial distribution of the emission.

\subsection{Modelling Diffuse X-ray/ $\gamma$-ray}

Figure 3 (bottom-left panel) presents broad band "diffuse" measurements. In the GeV range, Fermi/LAT did not confirm the EGRET excess, which was probably an instrumental effect [31]. Fermi/LAT is also a calorimeter providing improved cosmic-ray electron measurements. Taking into account this set of updated results leads to new GALPROP predictions that model quite accurately the emission in the Fermi/LAT energy range and which is also coherent with INTEGRAL/SPI measurements (Fig. 3). Nevertheless, the spectrum around $1 \mathrm{MeV}$ requires more investigation. This point is addressed in more details in [7].

While the solar modulation makes it difficult to measure the spectrum of low-energy electron, SPI can help to probe indirectly the electron (and positron) distributions in our Galaxy through their inverse Compton interactions.

\subsection{Summary}

The INTEGRAL observatory has lead to significant progress in the source census and this will continue. Hard X-ray/soft-gamma-ray surveys at energies below $100 \mathrm{keV}$ are currently being performed by IBIS/INTEGRAL and SWIFT/BAT. These surveys have ten times the number of sources (actually $\sim 1300$ ) found in surveys made in the previous decade. INTEGRAL/SPI enhances these at energies above $\sim 100 \mathrm{keV}$. Surveys in X-rays and $\gamma$-ray are also on-going. In X-rays the 2XMM catalogue ( with more than 250000 sources) has been issued. Fermi-LAT is performing the survey above $100 \mathrm{MeV}$ since 2008, and had detected 1451 sources [32]

Concerning the diffuse measurements, progress have been possible due to INTEGRAL, which shows clearly that point sources dominate the Milky-Way emission. SPI probes also indirectly the distribution of cosmic-rays electrons and positrons. In view of the large amounts of data accumulated with INTEGRAL/SPI, we are now able to constrain the spatial distribution of the diffuse emission above $100 \mathrm{keV}$. The derived "diffuse" interstellar emission distribution is qualitatively consistent with GALPROP code predictions.

Using Fermi, Planck, Wmap, Milagro and SPI together in addition to radio measurements will constrain the "diffuse" modelling on more than 20 decades in energy and expand our knowledge of the diffuse emission physics.

There are also perspectives for further advances in analysis methods, in particular a better description of source variability, which in turn may provide a better INTEGRAL/SPI census especially below $50 \mathrm{keV}$. INTEGRAL will still be operating for several more years, and the increased statistics will provide better diffuse maps and hence help in probing cosmic-ray electrons in our Galaxy. 


\section{References}

[1] Roques, J. P., Schanne, S., Von Kienlin, A., et al., 2003, A \& A , 411, L91

[2] Vedrenne, G., Roques, J. P., Schonfelder, V., et al., 2003, $A \&$ \& , 411, L63

[3] Jensen, P. L., Clausen, K., Cassi, C., et al., 2003, $A \&$ \& , 411, L7

[4] Scargle, D., 1998, ApJ , 504, 405

[5] Rouet, F. R., 2009, Partial computation of the inverse of a large sparse matrix-application to astrophysics,INP-ENSEEEIHT/IRIT, http://rouet.perso.enseeiht.fr/report.pdf

[6] Bouchet, L., et al., 2011, In prepration.

[7] Bouchet, L., 2011, In prepration.

[8] Bouchet, L., Jourdain, E., Roques, J. P, et al., 2008, ApJ , 679, 1315

[9] Revnivtsev, M. G., Sunyaev, R. A., Gilfanov, M. R., et al., 2004, AstL, 30, 527

[10] Bird, A. J., Bazzano, A., Bassani, L., et al., 2010, ApJ sup., 186, 1

[11] Krivonos, R., Tsygankov, S., Revnivtsev, M., et al., 2010, A \& A , 523, 61

[12] Cusumano, G., La Parola, V., Segreto, A., et al., 2010., $A \&$ \& , 524, 64

[13] Bazzano, A., Stephen, J. B., Fiocchi, M., et al., 2006, ApJ , 649, 9

[14] Bouchet, L., Roques, J. P., Mandrou, P., et al., 2005, ApJ , 635, 1103

[15] Jourdain, E., Roques, J. P. \& Bouchet, L., 2007, PThPS, 169, 221J

[16] Lebrun, F., Terrier, R., Bazzano, A., et al., 2004, Nature, 428, 293

[17] Revnivtsev, M., Sazonov, S., Gilfanov, M., et al., 2006, A \& A , 452, 169

[18] Krivonos, R., Revnivtsev, M., Churazov, E., et al., 2007, A\& A , 463, 957

[19] Strong, A. W., Porter, T A., Digel, et al., 2010, ApJ Letters, 722, L58

[20] Bouchet, L., Roques, J. P., \& Jourdain, E., 2010, ApJ , 720, 1772

[21] Weidenspointner, G., Skinner, G., Jean, P., et al., 2008, Nature, 451, 159

[22] Churazov, E., Sunyaev, R., Sazonov, S., et al., 2005, MNRAS, 337, 1377

[23] Jean, P., Knödlseder, J., Gillard, W., et al., 2006, $A \&$ \& A , 445, 579

[24] Higdon, J. C., Lingenfelter, R. E., \& Rothschild,R. E., 2009, ApJ , 698, 350

[25] Churazov, E., Sazonov, S., Ysygankov S., et al., 2010, MNRAS, arXiv:1010.0864C

[26] Smith, D. M., 2004, ESA-SP-552, 45

[27] Harris, M. J, Knödlseder, J., Jean, P., et al., 2005, $A$ \& A , 433, L49

[28] Wang, W., Harris, M. J., Diehl, R., et al., 2007, $A \&$ \& , 469, 1005

[29] Türler, M., Chernyakova, M., Courvoisier, T. J.-L., et al., 2010, A\& A , 512, 49

[30] Porter, T. A., Moskalenko, I. V., Strong, A. W., et al., 2008, ApJ , 682, 400

[31] Abdo, A. A., Ackermann, M., Ajello, M., et al., 2009, Phys. Rev. Lett., 103, 25, id. 251101

[32] Abdo, A. A., Ackermann, M., Ajello, M., et al., 2010, Phys. Rev. Lett., 104, 10, id. 10110

[33] Malzac, J., Petrucci, P. O., Jourdain, E., et al., 2006, $A \&$ \& A , 448, 1125 